\title{
Infinitely many hyperbolic Coxeter groups through dimension 19
}

\author{
DANIEL ALLCOCK
}

\begin{abstract}
We prove the following: there are infinitely many finite-covolume (resp. cocompact) Coxeter groups acting on hyperbolic space $H^{n}$ for every $n \leq 19$ (resp. $n \leq 6$ ). When $n=7$ or 8 , they may be taken to be nonarithmetic. Furthermore, for $2 \leq n \leq 19$, with the possible exceptions $n=16$ and 17, the number of essentially distinct Coxeter groups in $H^{n}$ with noncompact fundamental domain of volume $\leq V$ grows at least exponentially with respect to $V$. The same result holds for cocompact groups for $n \leq 6$. The technique is a doubling trick and variations on it; getting the most out of the method requires some work with the Leech lattice.
\end{abstract}

20F55; 51M20, 51M10

\section{Introduction}

The purpose of this paper is to prove the following theorems. Recall that a Coxeter polyhedron in hyperbolic space $H^{n}$ is the natural fundamental domain for a Coxeter group, ie, it is a convex polyhedron with all dihedral angles being integral submultiples of $\pi$.

Theorem 1.1 There are infinitely many isometry classes of finite-volume Coxeter polyhedra in $H^{n}$, for every $n \leq 19$. For $2 \leq n \leq 6$, they may be taken to be either compact or noncompact, and for $n=7$ or 8 , they may be taken to be either arithmetic or nonarithmetic.

Theorem 1.2 For every $n \leq 19$, with the possible exceptions of $n=16$ and 17 , the number of isometry classes of Coxeter polyhedra in $H^{n}$ of volume $\leq V$ grows at least exponentially with respect to $V$. For $2 \leq n \leq 6$, these polyhedra may be taken to be either compact or noncompact.

The essentially new results are the nonarithmetic examples, the noncompact cases of both theorems for $n \geq 9$, the compact case of Theorem 1.1 for $n=6$, and the compact case of Theorem 1.2 for $n=5$ and 6 . Makarov [10] exhibited infinitely many compact 
Coxeter polyhedra in $H^{n \leq 5}$, and the remaining parts of the theorems are relatively easy, using known right-angled polyhedra. While our results suggest that there is no hope for a complete enumeration of hyperbolic Coxeter polyhedra, several authors have classified certain interesting classes of polyhedra, eg, Esselmann [8], Kaplinskaja [9] and Tumarkin $[16 ; 15 ; 17]$.

The only dimension $n$ for which a finite-volume Coxeter polyhedron in $H^{n}$ is known, and in which it remains unknown whether there are infinitely many, is $n=21$, an example due to Borcherds [1]. The corresponding $n$ for compact polyhedra are $n=7$ and 8 , by examples of Bugaenko [3;5]. Therefore our results may be close to optimal, although we expect that the hypothesis $n \neq 16,17$ of Theorem 1.2 can be removed and that better results for nonarithmetic groups hold. On the other hand, there is still a considerable gap between the dimensions in which Coxeter polyhedra are known to exist and those in which they are known not to exist. Namely, Vinberg [19] proved that there are no compact Coxeter polyhedra in $H^{n \geq 30}$, and Prokhorov [13] proved the absence of finite-volume Coxeter polyhedra in $H^{n \geq 996}$.

The heart of our construction is a simple doubling trick. We call a wall of a Coxeter polyhedron $P$ a doubling wall if the angles it makes with the walls it meets are all even submultiples of $\pi$. By the double of $P$ across one of its walls we mean the union of $P$ and its image under reflection across the wall. We call a polyhedron redoublable if it is a Coxeter polyhedron with two doubling walls that do not meet each other in $H^{n}$.

Lemma 1.3 The double of a Coxeter polyhedron $P$ across a doubling wall is a Coxeter polyhedron. If the doubling wall is disjoint from another doubling wall, so that $P$ is redoublable, then the double is also redoublable.

To construct infinitely many compact (resp. finite-volume) Coxeter polyhedra in $H^{n}$ it now suffices to find a single compact (resp. finite-volume) redoublable polyhedron in $H^{n}$ : double it, then double the double, and so on.

Many already-known Coxeter polyhedra happen to be redoublable; in fact, to prove Theorem 1.1 we only need to produce a few examples. We do this in Section 2, where we give a fairly uniform proof of the existence of finite-volume redoublable polyhedra in every dimension $\leq 19$. We do this without having to compute the details of their Coxeter diagrams.

We provide the diagrams in Section 3, for completeness and also for use in Section 4, where we discuss variations on the doubling construction and establish Theorem 1.2. We also show that the Coxeter group of a redoublable polyhedron contains subgroups of every positive index that are themselves Coxeter groups. 
For the most part we follow Vinberg [18] regarding notation and terminology. A wall of a polyhedron is a codimension one face. We say that two walls meet if they have nonempty intersection in $H^{n}$. If they do not meet, and their closures in $H^{n} \cup S_{\infty}^{n-1}$ have a common ideal point, we call them parallel. If they do not share even an ideal point then we call them ultraparallel. Because the terms 'vertices' and 'edges' play many roles, we refer to the vertices and edges of a Coxeter diagram as nodes and bonds. We join two nodes by no bond (resp. a single bond or double bond) if the corresponding walls make an angle of $\pi / 2$ (resp. $\pi / 3$ or $\pi / 4$ ), and by a heavy (resp. dashed) bond if the walls are parallel (resp. ultraparallel). For other angles $\pi / n$ we would draw a single bond and mark it with the numeral $n$. We call a Coxeter diagram spherical if its Coxeter group is finite, because finite Coxeter groups act naturally on spheres. When $X$ is a polyhedron or a Coxeter diagram we write $W(X)$ for the associated Coxeter group, or just $W$ when the meaning is clear. By a set of simple roots for a polyhedron in $H^{n} \subseteq P\left(\mathbb{R}^{n, 1}\right)$, we mean a set of vectors $r_{i} \in \mathbb{R}^{n, 1}$ with positive norms and nonpositive inner products, with the hyperplanes $r_{i}^{\perp}$ defining the walls.

We refer to a tip of a $D_{n}$ or $E_{n}$ diagram as an ear if it lies at distance 1 from the branch point and as a tail if it lies at maximal distance from the branch point. Explicitly: $D_{n>4}$ has two ears and a tail, $E_{7}$ and $E_{8}$ each have one ear and one tail, $E_{6}$ has an ear and two tails, and $D_{4}$ has three tails which are also ears.

I am grateful to the referee for the reference to Ruzmanov [14], to Vadim Bugaenko for allowing me to present unpublished details from his thesis and to Anna Felikson for her reference to Bugaenko [4] and her helpful suggestions, including one which led to the current proof of Theorem 1.2. My original proof was extremely intricate and not very conceptual. I used the PARI/GP system [11] for some of the calculations. I am grateful to the National Science Foundation for supporting this research with grant DMS-0245120.

\section{Construction of redoublable polyhedra}

We begin with the proof of Lemma 1.3, and survey some polyhedra in the literature that are redoublable. Then we give a systematic method for looking for redoublable polyhedra as faces of known Coxeter polyhedra, and provide many examples. The construction is 'soft' in the sense that we can prove our examples exist without needing to understand very much about them. See the next section for the diagrams.

Proof of Lemma 1.3 We write $w$ for the doubling wall and $2 P$ for the double of $P$ across $w$. Every dihedral angle of $2 P$ is either a dihedral angle of $P$ or twice a 
dihedral angle of $P$ involving $w$. The former are integer submultiples of $\pi$ because $P$ is a Coxeter polyhedron, and the latter are also because the dihedral angles involving $w$ have the form $\pi /$ (an even integer). Therefore $2 P$ is a Coxeter polyhedron. For its redoublability, observe that the second doubling wall and its reflection across $w$ are disjoint doubling walls of $2 P$.

The simplest redoublable polyhedra in the literature have all dihedral angles equal to $\pi / 2$; these are called right-angled polyhedra. Compact examples are known to exist in $H^{n}$ for $n \leq 4$ and finite-volume ones for $n \leq 8$. See Potyagailo and Vinberg [12] for these examples and also for a proof that compact (resp. finite-volume) examples cannot exist for $n>4$ (resp. $n>14$ ).

Vinberg [18] and Vinberg-Kaplinskaja [21] found Coxeter groups acting on $H^{n \leq 19}$ by considering the Weyl chamber (we call it $P_{n}$ ) for the reflection subgroup of the isometry group of the lattice $I_{n, 1}$, ie, the integer quadratic form

$$
-x_{0}^{2}+x_{1}^{2}+\cdots+x_{n}^{2} .
$$

By definition $P_{n}$ is a Coxeter polyhedron, and for $n \leq 19$ it has finite volume; its Coxeter diagram appears in [18] for $n \leq 17$ and in [21] for $n=18$ or 19. It turns out that $P_{n}$ is redoublable for $n=2$ (walls 2 and 3), $n=10$ (walls 10 and 12), $n=14$ (walls 14 and 17), $n=16$ (walls 16 and 20), $n=17$ (walls 17 and 21), $n=18$ and $n=19$. The specified walls are disjoint doubling walls, and refer to the figures on p. 32 of [18]. Figures $1 \beta$ and $1 \gamma$ of [21] display $3+12=15$ pairwise disjoint doubling walls of $P_{18}$, and figure $2 \gamma$ displays 20 pairwise disjoint doubling walls of $P_{19}$.

Vinberg also found the Weyl chamber for the reflection subgroup of the isometry group of the integer quadratic form

$$
-2 x_{0}^{2}+x_{1}^{2}+\cdots+x_{n}^{2}
$$

for $n \leq 14$. It turns out to be redoublable for $n=2$ (walls 1 and 3), $n=3$ (walls 3 and 5), $n=9$ (walls 9 and 12, or 10 and 12), $n=10$ (walls 11 and 13), $n=11$ (walls 11 and 15), $n=13$ (walls 13 and 18, or 13 and 19, or 14 and 18, or 14 and 19) and $n=14$ (walls 15 and 20). The wall numbering refers to [18, page 34].

Bugaenko [4] investigated the reflection group of the quadratic form

$$
-(1+\sqrt{2}) x_{0}^{2}+x_{1}^{2}+\cdots+x_{n}^{2}
$$

over $\mathbb{Z}[\sqrt{2}]$, and found that it has compact fundamental domain if and only if $n \leq 6$. For $n=3,4,5$ and 6 the polyhedra are redoublable. For $n \leq 5$ the diagrams appear in [4]. (There are some minor typographical errors in the node-labeling for $n=5$.) 


\begin{tabular}{|c|c|c|c|c|c|c|c|c|c|c|c|c|c|c|}
\hline & $a_{0}$ & $b_{0}$ & $a_{1}$ & $b_{1}$ & $a_{2}$ & $b_{2}$ & $a_{3}$ & $b_{3}$ & $a_{4}$ & $b_{4}$ & $a_{5}$ & $b_{5}$ & $a_{6}$ & $b_{6}$ \\
\hline$r_{1}$ & 0 & 0 & -1 & 0 & 1 & 0 & 0 & 0 & 0 & 0 & 0 & 0 & 0 & 0 \\
\hline$r_{2}$ & 0 & 0 & 0 & 0 & -1 & 0 & 1 & 0 & 0 & 0 & 0 & 0 & 0 & 0 \\
\hline$r_{3}$ & 0 & 0 & 0 & 0 & 0 & 0 & -1 & 0 & 1 & 0 & 0 & 0 & 0 & 0 \\
\hline$r_{4}$ & 0 & 0 & 0 & 0 & 0 & 0 & 0 & 0 & -1 & 0 & 1 & 0 & 0 & 0 \\
\hline$r_{5}$ & 0 & 0 & 0 & 0 & 0 & 0 & 0 & 0 & 0 & 0 & -1 & 0 & 1 & 0 \\
\hline$r_{6}$ & 0 & 0 & 0 & 0 & 0 & 0 & 0 & 0 & 0 & 0 & 0 & 0 & -1 & 0 \\
\hline$r_{7}$ & 1 & 0 & 1 & 1 & 0 & 0 & 0 & 0 & 0 & 0 & 0 & 0 & 0 & 0 \\
\hline$r_{8}$ & 1 & 1 & 1 & 1 & 1 & 1 & 1 & 1 & 0 & 0 & 0 & 0 & 0 & 0 \\
\hline$r_{9}$ & 2 & 1 & 1 & 1 & 1 & 1 & 1 & 1 & 1 & 1 & 1 & 1 & 1 & 0 \\
\hline$r_{10}$ & 2 & 1 & 2 & 1 & 1 & 1 & 1 & 1 & 1 & 1 & 1 & 0 & 0 & 0 \\
\hline$r_{11}$ & 3 & 2 & 3 & 2 & 3 & 2 & 1 & 1 & 1 & 1 & 1 & 1 & 0 & 0 \\
\hline$r_{12}$ & 2 & 2 & 2 & 2 & 2 & 1 & 1 & 1 & 1 & 1 & 1 & 1 & 1 & 1 \\
\hline$r_{13}$ & 4 & 2 & 3 & 2 & 3 & 2 & 2 & 2 & 2 & 1 & 1 & 1 & 1 & 1 \\
\hline$r_{14}$ & 7 & 5 & 7 & 5 & 6 & 4 & 4 & 3 & 3 & 2 & 3 & 2 & 2 & 1 \\
\hline$r_{15}$ & 4 & 3 & 4 & 3 & 3 & 2 & 3 & 2 & 2 & 1 & 2 & 1 & 1 & 1 \\
\hline$r_{16}$ & 4 & 3 & 4 & 3 & 4 & 3 & 2 & 1 & 1 & 1 & 1 & 1 & 1 & 1 \\
\hline$r_{17}$ & 6 & 4 & 5 & 4 & 5 & 4 & 3 & 2 & 3 & 2 & 2 & 2 & 2 & 1 \\
\hline$r_{18}$ & 6 & 4 & 6 & 4 & 5 & 4 & 3 & 2 & 2 & 2 & 2 & 1 & 2 & 1 \\
\hline$r_{19}$ & 8 & 5 & 7 & 5 & 7 & 5 & 3 & 3 & 3 & 2 & 3 & 2 & 3 & 2 \\
\hline$r_{20}$ & 8 & 5 & 8 & 5 & 7 & 5 & 3 & 2 & 3 & 2 & 3 & 2 & 2 & 2 \\
\hline$r_{21}$ & 8 & 6 & 8 & 5 & 7 & 5 & 5 & 3 & 4 & 3 & 4 & 3 & 2 & 1 \\
\hline$r_{22}$ & 6 & 4 & 6 & 4 & 5 & 3 & 3 & 2 & 3 & 2 & 3 & 2 & 1 & 1 \\
\hline$r_{23}$ & 6 & 4 & 6 & 4 & 5 & 4 & 3 & 2 & 3 & 2 & 1 & 1 & 1 & 1 \\
\hline$r_{24}$ & 6 & 5 & 6 & 4 & 6 & 4 & 4 & 3 & 3 & 2 & 3 & 2 & 1 & 1 \\
\hline$r_{25}$ & 10 & 7 & 9 & 7 & 9 & 6 & 5 & 4 & 5 & 4 & 3 & 2 & 3 & 2 \\
\hline$r_{26}$ & 10 & 8 & 10 & 7 & 10 & 7 & 6 & 4 & 5 & 4 & 3 & 2 & 2 & 2 \\
\hline$r_{27}$ & 8 & 6 & 8 & 6 & 7 & 5 & 5 & 3 & 3 & 2 & 3 & 2 & 3 & 2 \\
\hline$r_{28}$ & 12 & 9 & 12 & 8 & 11 & 8 & 7 & 5 & 5 & 3 & 5 & 3 & 4 & 3 \\
\hline$r_{29}$ & 10 & 7 & 10 & 7 & 8 & 6 & 6 & 4 & 5 & 3 & 3 & 2 & 3 & 2 \\
\hline$r_{30}$ & 10 & 7 & 10 & 7 & 9 & 6 & 5 & 3 & 4 & 3 & 4 & 3 & 3 & 2 \\
\hline$r_{31}$ & 14 & 10 & 14 & 10 & 12 & 8 & 8 & 5 & 7 & 5 & 5 & 3 & 4 & 3 \\
\hline$r_{32}$ & 10 & 8 & 10 & 7 & 10 & 7 & 6 & 4 & 4 & 3 & 4 & 3 & 3 & 2 \\
\hline$r_{33}$ & 12 & 8 & 12 & 8 & 10 & 7 & 6 & 4 & 6 & 4 & 4 & 3 & 3 & 2 \\
\hline$r_{34}$ & 12 & 9 & 12 & 8 & 11 & 8 & 7 & 5 & 6 & 4 & 4 & 3 & 3 & 2 \\
\hline
\end{tabular}

Table 2.1: Simple roots for Bugaenko's polyhedron in $H^{6}$. Each root has coordinates $\left(a_{0}+b_{0} \sqrt{2}, \ldots, a_{6}+b_{6} \sqrt{2}\right)$. 


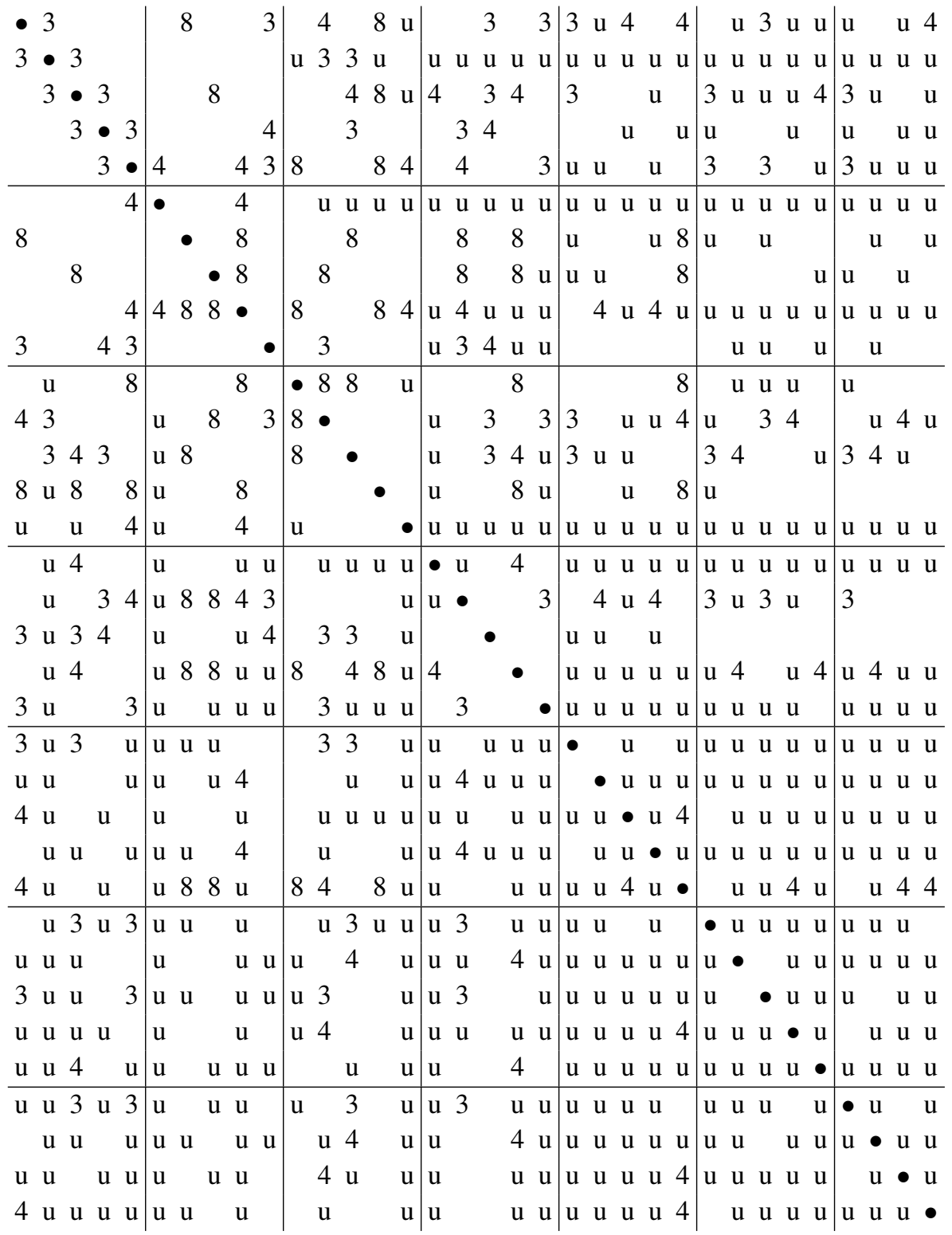

Table 2.2: Bond-labels of the Coxeter diagram for Bugaenko's polyhedron in $H^{6}$. A blank indicates an bond-label of 2 (orthogonality), and ' $u$ ' indicates ultraparallelism. 
For $n=6$, Bugaenko computed the polyhedron but did not describe it completely. We are grateful to him for providing the details, which we will need in Section 4. His set of simple roots appears in table 2.1, and the matrix of bond-labels of the Coxeter diagram appears in table 2.2. Entries that would be 2's have been left blank. It is easy to check redoublability, eg, by considering walls 9 and 19. We remark that Bugaenko also obtained redoublable polyhedra in $H^{5}$ and $H^{6}$ in his study [3] of polyhedra over $\mathbb{Z}[(1+\sqrt{5}) / 2]$.

Our method resembles the construction by Ruzmanov [14] of finite-volume nonarithmetic Coxeter polyhedra in $H^{6}, \ldots, H^{10}$; his examples in $H^{7}$ and $H^{8}$ are redoublable. His construction involves gluing two polyhedra to get a larger polyhedron, and then "cutting off corners" by hyperplanes. Cutting off a corner creates a doubling wall. In $H^{7}$ and $H^{8}$, he cuts off two corners, leading to redoublable polyhedra. We expect nonarithmetic redoublable polyhedra to exist in some other dimensions, but we have not attempted a systematic study.

Because of these examples, to prove Theorem 1.1 we need only exhibit finite-volume redoublable polyhedra in $H^{12}$ and $H^{15}$. Nevertheless, we will work in all dimensions $\leq$ 19 , since our constructions are not very sensitive to dimension. Our examples rely on the following result of Borcherds [2, example 5.6].

Theorem 2.1 Suppose $P$ is a Coxeter polyhedron with diagram $\Delta$, and $p$ is the face corresponding to a spherical subdiagram $\sigma$ of $\Delta$ that has no $A_{n}$ or $D_{5}$ component. Then $p$ is itself a Coxeter polyhedron.

We will need more precise information about the shape of $p$, so we discuss how to obtain the Coxeter diagram of $p$ from that of $P$. These calculations provide a geometric proof of Borcherds' theorem.

Because the faces of $P$ are in bijection with the spherical subdiagrams of $\Delta$, the walls of $p$ correspond to the nodes $A$ of $\Delta$ which extend $\sigma$ to a larger but still-spherical diagram. We call such a node a spherical extension of $\sigma$. We say that a node of $\Delta$ attaches to $\sigma$ if it is joined to some node of $\sigma$ by an bond of any type. If $\sigma$ is as in Theorem 2.1 and $A$ is a spherical extension of it, then $A$ joins to at most one node of $\sigma$, and if it joins to a node of $\sigma$ then the bond is a single bond. (Because $\sigma$ has no $A_{n}$ components, any other extension of $\sigma$ would be non-spherical.) If $a$ and $b$ are two walls of $p$, coming from walls $A$ and $B$ of $P$, ie, $a=A \cap p$ and $b=B \cap p$, then their dihedral angle $\angle a b$ will be at most $\angle A B$. The new dihedral angles can be worked out by the following rules.

Theorem 2.2 Under the hypotheses of Theorem 2.1: 
(1) If neither $A$ nor $B$ attaches to $\sigma$, then $\angle a b=\angle A B$.

(2) If just one of $A$ and $B$ attaches to $\sigma$, say to the component $\sigma_{0}$, then

(a) if $A \perp B$ then $a \perp b$;

(b) if $A$ and $B$ are singly joined and adjoining $A$ and $B$ to $\sigma_{0}$ yields a diagram $B_{k}\left(\right.$ resp. $D_{k}, E_{8}$ or $\left.H_{4}\right)$ then $\angle a b=\pi / 4(\operatorname{resp} . \pi / 4, \pi / 6$ or $\pi / 10)$;

(c) otherwise, $a$ and $b$ do not meet.

(3) If $A$ and $B$ attach to different components of $\sigma$, then

(a) if $A \perp B$ then $a \perp b$;

(b) otherwise, $a$ and $b$ do not meet.

(4) If $A$ and $B$ attach to the same component of $\sigma$, say $\sigma_{0}$, then

(a) if $A$ and $B$ are unjoined and $\sigma_{0} \cup\{A, B\}$ is a diagram $E_{6}$ (resp. $E_{8}$ or $F_{4}$ ) then $\angle a b=\pi / 3(\operatorname{resp} . \pi / 4$ or $\pi / 4)$;

(b) otherwise, $a$ and $b$ do not meet.

Proof All conclusions that $a$ and $b$ do not meet are justified by observing that adjoining both $A$ and $B$ to $\sigma$ yields a non-spherical diagram. For the remaining cases we choose simple roots $r_{1}, \ldots, r_{\ell}$ for the nodes comprising $\sigma$. We write $Y$ for the span of $r_{1}, \ldots, r_{\ell}$ and $\Pi$ (resp. $\Pi^{\perp}$ ) for orthogonal projection in $\mathbb{R}^{n, 1}$ to $Y$ (resp. $Y^{\perp}$ ). If $s$ and $t$ are simple roots for $P$ corresponding to $A$ and $B$, then $\Pi^{\perp}(s)$ and $\Pi^{\perp}(t)$ are simple roots for $p$ corresponding to $a$ and $b$. If neither $A$ nor $B$ joins to $\sigma$ then $s$ and $t$ are their own projections to $Y^{\perp}$, and $\angle a b=\angle A B$, justifying (1). More generally, the norms and inner product of $\Pi^{\perp}(s)$ and $\Pi^{\perp}(t)$ determine $\angle a b$. We have $\left(\Pi^{\perp}(s)\right)^{2}=s^{2}-\Pi(s)^{2}$ and similarly for $t$, and $\Pi^{\perp}(s) \cdot \Pi^{\perp}(t)=s \cdot t-\Pi(s) \cdot \Pi(t)$, so it suffices to find the norms and inner product of $\Pi(s)$ and $\Pi(t)$. We may introduce whatever coordinates we like to describe the $r_{i}$, and determine $\Pi(s)$ and $\Pi(t)$ in terms of these coordinates by using their known inner products with the $r_{i}$. With $\Pi(s)$ and $\Pi(t)$ in hand, it is easy to compute $\angle a b=\pi-\angle\left(\Pi^{\perp}(s), \Pi^{\perp}(t)\right)$.

Unless $A$ and $B$ attach to the same component of $\sigma$ we have $\Pi(s) \perp \Pi(t)$, in which case $s \perp t$ implies $\Pi^{\perp}(s) \perp \Pi^{\perp}(t)$. This justifies (2)(a) and (4)(a).

In all remaining cases, enlarging $\sigma_{0}$ to $\sigma_{0} \cup\{A, B\}$ is one of the extensions $B_{k} \rightarrow B_{k+2}$, $D_{k} \rightarrow D_{k+2}, B_{2} \rightarrow F_{4}, D_{4} \rightarrow E_{6}, D_{6} \rightarrow E_{8}, E_{6} \rightarrow E_{8}$ and $I_{2}(5) \rightarrow H_{4}$; these must be worked out one by one. As an example, we treat the case where $\sigma_{0}$ is a $D_{6}$, $A$ and $B$ are unjoined, $A$ attaches to an ear of the $D_{6}$ and $B$ to the tail. We take the standard model of the $D_{6}$ root system in $\mathbb{R}^{6}$ :

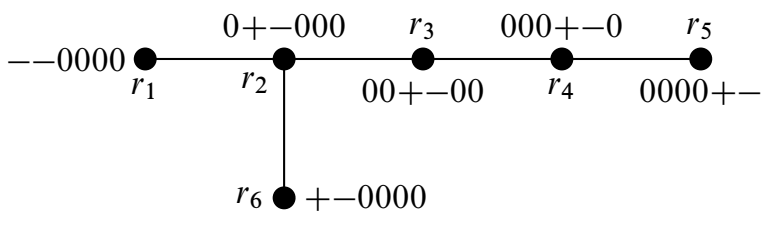


where + and - indicate 1 and -1 . We take $s$ and $t$ to have norm 2, with $s \cdot r_{1}=-1$ and $t \cdot r_{5}=-1$, and their inner products with the other $r_{i}$ being 0 . Then $\Pi(s)$ must be the vector $\frac{1}{2}(1,1,1,1,1,1)$ and $\Pi(t)$ the vector $(0,0,0,0,0,1)$. These have norms $3 / 2$ and 1 , so $\Pi^{\perp}(s)$ and $\Pi^{\perp}(t)$ have norms $1 / 2$ and 1 . Also, $\Pi^{\perp}(s) \cdot \Pi^{\perp}(t)=$ $s \cdot t-\Pi(s) \cdot \Pi(t)=0-1 / 2$, and we get $\angle a b=\pi / 4$. The other calculations are similar; for convenient models of the root systems see for example [7, Chapter 4]. We remark that simple roots for $I_{2}(5)$ consist of two norm 2 vectors with inner product $-\phi$, where $\phi=(1+\sqrt{5}) / 2$ is the golden ratio.

\section{Remarks}

(1) Borcherds formulated Theorem 2.1 using the Tits cone rather than hyperbolic space, so that it applies in any Coxeter group; Theorem 2.2 extends similarly.

(2) For hyperbolic polyhedra it is natural to distinguish between parallelism and ultraparallelism of walls of $p$ which do not meet. This refinement may be obtained by extending the above rules as follows. Suppose $a$ and $b$ do not meet. If adjoining both $A$ and $B$ to $\sigma$ yields a diagram with an affine component, then $a$ and $b$ are parallel; otherwise, $a$ and $b$ are ultraparallel.

For a less-complicated statement, we isolate the conclusions of Theorem 2.2 that we will use in our examples. The proof consists of chasing through the various cases of the theorem.

Corollary 2.3 Suppose $P, \Delta, p$ and $\sigma$ are as in Theorem 2.2. Suppose $w$ is a wall of $p$ corresponding to a spherical extension of $\sigma$ which attaches to some $D_{n \geq 6}, E_{6}$ or $E_{7}$ component of $\sigma$. Then $w$ is a doubling wall of $p$. Two such extensions of the same component of $\sigma$ yield disjoint doubling walls, except in the case that adjoining both of them to $\sigma$ enlarges that component by $D_{6} \rightarrow E_{8}$.

Our examples take $P$ to be Conway's infinite-volume Coxeter polyhedron in $H^{25}$; see [7, Chapter 27]. This has diagram $\Delta$ with infinitely many nodes, one for each element of the Leech lattice $\Lambda \subseteq \mathbb{R}^{24}$. Two nodes are joined by no bond (resp. a single bond, a heavy bond, or a dashed bond) if the difference of the lattice vectors has norm 4 (resp. 6,8 , or more than 8 ). To visualize $P$, regard $\Lambda$ as a subset of $\mathbb{R}^{24} \subseteq \partial H^{25}$ in the upper-half-space model for $H^{25}$. Consider the hyperplanes which appear in this model as hemispheres of radius $\sqrt{2}$ centered at lattice points. The region above the hyperplanes is $P$, and the angles between its walls can be worked out by elementary geometry and seen to agree with our description. Because the Coxeter diagram essentially is the Leech lattice, we write $\Lambda$ in place of $\Delta$. 
The covering radius of $\Lambda$ is $\sqrt{2}$, so the hemispheres exactly cover $\mathbb{R}^{24} \subseteq \partial H^{25}$. This implies that every face of dimension $>1$ except $P$ itself has finite volume; for a formal proof see [1, Lemma 4.3]. The isometry group of $P$ is the infinite group $C o_{\infty}$ of all isometries of $\Lambda$, including translations. The idea of studying the faces of $P$ is due to Conway and Sloane [6] and was refined by Borcherds [1].

Example 2.4 Finite-volume redoublable polyhedra in $H^{19}$ and $H^{18}$ : By the calculations required to prove Theorem 24 (resp. Theorem 22) in [7, Chapter 23], $\Lambda$ contains a single orbit of diagrams $E_{6}$ (resp. $E_{7}$ ); such a diagram has three extensions to $E_{7}$ (resp. two extensions to $E_{8}$ ). (Note that [7, Chapter 23] uses nonstandard notation, writing $e_{n}$ for $E_{n}, E_{n}$ for $\widetilde{E}_{n}$ and similarly for $A_{n}$ and $D_{n}$.) Therefore the faces of $P$ corresponding to the $E_{6}$ and $E_{7}$ diagrams are redoublable. The $E_{6}$ face was found by Vinberg [20] and interpreted as such by Borcherds [1], who also found the $E_{7}$ face. These faces are simpler than the $D_{6}$ and $D_{7}$ faces of the next example, having only 36 and 24 walls, rather than 50 and 37.

Example 2.5 Finite-volume redoublable polyhedra in $H^{19}, \ldots, H^{16}: \Lambda$ contains affine diagrams $\widetilde{D}_{7}, \ldots, \widetilde{D}_{10}$; for explicit vectors see figs. 23.14, 23.24, 23.16 and 23.25 of [7, Chapter 23]. Therefore, $\Lambda$ contains for each $n=6, \ldots, 9$ a $D_{n}$ that has two distinct extensions to a $D_{n+1}$. By the corollary, these $D_{n}$ faces of $P$ are redoublable. These examples turn out to be the polyhedra $P_{25-n}$ of Vinberg and Vinberg-Kaplinskaja; see [1]. (The $D_{4}$ face is Borcherds' Coxeter polyhedron; it is not redoublable because of the $\pi / 3$ appearing in case (4)(a) of Theorem 2.2.)

For the cases $n=6$ or 7 there is a special phenomenon, because the $D_{n}$ admits spherical extensions to $E_{n+1}$ as well as to $D_{n+1}$. Therefore one expects a $D_{6}$ or $D_{7}$ face of a Coxeter polyhedron to have unusually many doubling walls, and be unusually likely to be redoublable. This suggested looking at $D_{6} D_{n}$ and $D_{7} D_{n}$ faces of $P$, which led to the examples below.

Example 2.6 Finite-volume redoublable polyhedra in $H^{15}$ and $H^{14}$ : We consider faces $D_{6} D_{4}$ and $D_{7} D_{4}$ of $P$. By the calculations leading to figure 23.20 of [7, Chapter 23], $C o_{\infty}$ acts transitively on $D_{4}$ 's in $\Lambda$, and the elements of $\Lambda$ not joined to $D_{4}$ form the incidence graph of the points and lines of $\mathbf{P}^{2}\left(\mathbf{F}_{4}\right)$. It is easy to find a $D_{7}$ subdiagram of this graph that has two distinct extensions to $E_{8}$. Therefore the $D_{7} D_{4}$ face is redoublable. Discarding the tail of the $D_{7}$, the extensions $D_{7} \rightarrow E_{8}$ become extensions $D_{6} \rightarrow E_{7}$ and the same argument shows that the $D_{6} D_{4}$ face is also redoublable. 
Example 2.7 Finite-volume redoublable polyhedra in $H^{13}$ and $H^{12}$ : We consider faces $D_{6} D_{6}$ and $D_{6} D_{7}$ of $P$. By the calculations leading to figure 23.20 of [7, Chapter 23], $C o_{\infty}$ acts transitively on $D_{6}$ 's, and the elements of $\Lambda$ not joined to a $D_{6}$ form the graph which is the first barycentric subdivision of the Petersen graph. One proceeds exactly as in the previous example, finding a $D_{7}$ subgraph having two extensions to $E_{8}$.

Example 2.8 Finite-volume redoublable polyhedra in $H^{n}$ for $n=14$ and $n=$ $12, \ldots, 2$ : We seek a suitable face $D_{7} D_{n}$ of $P$, namely one having two extensions to $E_{8} D_{n}$ and/or $D_{8} D_{n}$; such extensions will yield doubling walls of the face, necessarily disjoint. We could proceed by considering each $D_{n}$ in turn, looking for $D_{7}$ 's not joined to it. But it is easier to fix an affine diagram $\widetilde{E}_{8}$ and find a $D_{n}$ disjoined from it, for $n=4$ and $n=6, \ldots, 16$. Then the two extensions $D_{7} \rightarrow D_{8}$ and $D_{7} \rightarrow E_{8}$ inside $\widetilde{E}_{8}$ show that the $D_{7} D_{n}$ face is redoublable. We don't even need to look for such an $\widetilde{E}_{8}$ since Conway, Parker and Sloane give explicit vectors forming an $\widetilde{E}_{8} \widetilde{D}_{16}$; see [7, figure 23.27]. We have already seen the $n=4$ and $n=6$ cases in examples 2.6 and 2.7.

\section{Explicit Diagrams}

In this section we give the Coxeter diagrams for the redoublable polyhedra from examples 2.6-2.8 of Section 2. They are all faces of the $D_{6}$ face of Conway's polyhedron $P$, so we begin by describing the 50 spherical extensions of $D_{6}$ in $\Lambda$. These define the polyhedron $P_{19}$ of Vinberg and Kaplinskaja, which is completely described in [21]; all we do is introduce a notation that allows easier record-keeping and makes the $S_{5}$ symmetry manifest.

Conway, Parker and Sloane [7, pages 495-496] choose specific elements of $\Lambda$ forming a $D_{6}$, which they call $\varnothing,[\widehat{\mathrm{I}}],[\widehat{\mathrm{II}}],[\widehat{\mathrm{III}}],[C]$ and $[\infty]$. The ears are $[\widehat{\mathrm{II}}]$ and $[\widehat{\mathrm{II}}]$ and the tail is $[\infty]$. To name the elements of $\Lambda$ extending $D_{6}$ to $D_{6} A_{1}$ and to $D_{7}$, they refer to a set $C=\{\infty, 0,1,2,3,4\}$. They label the $10+15$ extensions to $D_{6} A_{1}$ by the 10 duads (two-point sets) not containing $\infty$ and the 15 synthemes (a syntheme is a partition of $C$ into three duads). They label the five $D_{7}$ extensions by the duads containing $\infty$. The setwise stabilizer of $D_{6}$ in $C o_{\infty}$ is $S_{5}$, realized as the group of permutations of $C$ fixing $\infty$. The odd elements of $S_{5}$ exchange the ears of $D_{6}$.

They do not name the 20 extensions to $E_{7}$, so we introduce symbols $a b \mid c d e$ where $a, \ldots, e$ are $0, \ldots, 4$ in any order, with two such symbols considered equivalent if they differ by a cyclic permutation of the terms after the bar, or by a simultaneous 
application of a transposition after the bar and reversal of the terms before the bar. That is,

$$
a b|c d e=a b| e c d=a b|d e c=b a| e d c=b a|d c e=b a| c e d .
$$

We extend Sylvester's duad/syntheme language by calling such an equivalence class a dryad. The term comes from combining 'duad' and 'triad' and observing that the result is a misspelling of an existing English word.

Conway, Parker and Sloane give explicit elements of $\Lambda$ represented by their duads and synthemes. To describe the element of $\Lambda$ represented by a dryad $a b \mid c d e$, we refer to figure 23.18 of [7], which names the positions of the $4 \times 6$ MOG array, which is used for organizing the 24 coordinates. Begin with all coordinates 0 , then place 2 's in the spots marked by $c, d, e, I$ and by the synthemes

$$
\infty c . a d . b e, \infty e . a c . b d \text {, and } \infty \text { d.ae.bc. }
$$

One must check that these instructions respect the equivalences among the symbols $a b \mid c d e$. Finally, place a 2 in whichever one of the spots II and III yields an element of $\Lambda$. See [7, Chapter 11] for how to carry out this calculation. $S_{5}$ acts on the dryads by permuting $\{0, \ldots, 4\}$.

With all 50 extensions of $D_{6}$ given by explicit elements of $\Lambda$, one can work out the joins in the diagram $\Lambda$; the $S_{5}$ symmetry makes this fairly easy. The only joins among duads and synthemes are that each syntheme is joined to the three duads comprising it. A dryad $a b \mid c d e$ is joined to the duads $a b, \infty a, \infty b$ and to the synthemes of (3-1). The dryads fall into two orbits under $A_{5} \subseteq S_{5}$, corresponding to which ear of $D_{6}$ they join. The dryad $01 \mid 234$ joins to [ $\widehat{\mathrm{III}}]$ and the other dryads join to [ $\widehat{\mathrm{II}}]$ or [ $[\widehat{\mathrm{III}}]$ according to whether they differ from $01 \mid 234$ by an odd or even permutation. Two dryads are joined just if they lie in different $A_{5}$ orbits and their duads are disjoint. That is,

$$
a b|c d e=a b| e c d=a b \mid d e c
$$

is joined to the dryads

$$
d c|a b e \quad, \quad c e| a b d \text { and } e d \mid a b c
$$

and no others. A cute way to express the joins among the dryads is that they form a double cover of the Petersen graph (the cover in which all circuits have even length).

Derivation of the diagrams for the polyhedra of examples 2.6-2.8 is now a lengthy record-keeping exercise. As explained above, our starting point is the $D_{6}$ face consisting of $\varnothing,[\widehat{\mathrm{I}}],[\widehat{\mathrm{II}}],[\widehat{\mathrm{III}}],[C]$ and $[\infty]$, the tail being [ $\infty]$. We extend it to $D_{6} D_{4}$ by taking the $D_{4}$ consisting of the duad 23 and its neighboring synthemes, and to $D_{6} D_{6}$ by adjoining 01 and $01.24 .3 \infty$. We extend these two diagrams to $D_{7} D_{4}$ and $D_{7} D_{6}$ 

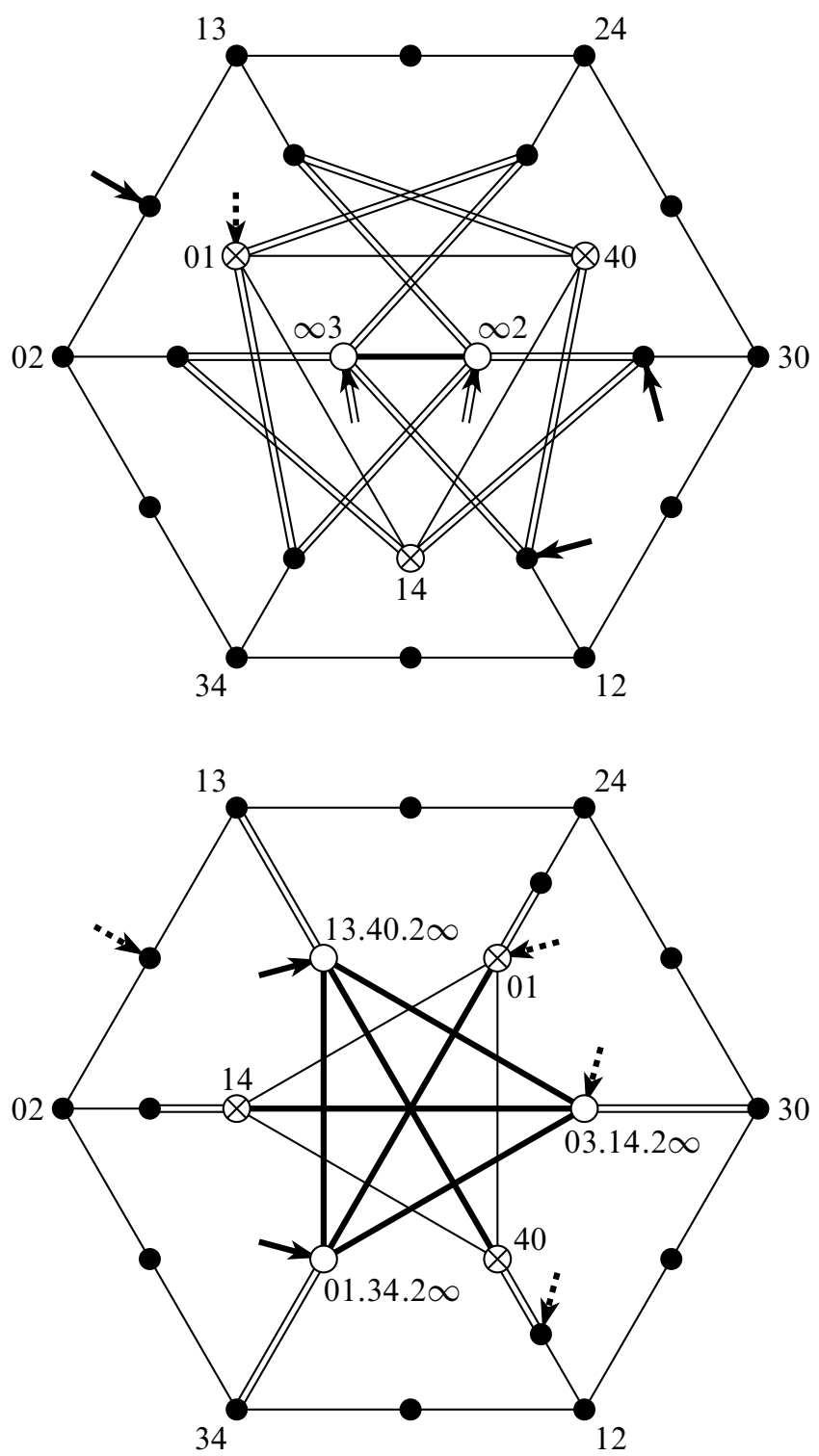

Figure 3.1: The $D_{6} D_{4}$ and $D_{7} D_{4}$ faces. The arrows show the joins of $01 \mid 234$, an $E_{7} D_{4}$ (or $E_{8} D_{4}$ ) extension. The other 5 dryads and their joins to the diagram are got by applying diagram automorphisms; any two dryads are joined by a dashed line. The permutations (410) and (14) act on the outer hexagon by $120^{\circ}$ rotation and by top-to-bottom reflection. The first figure has an extra symmetry, (14)(23), acting by left-to-right reflection. 

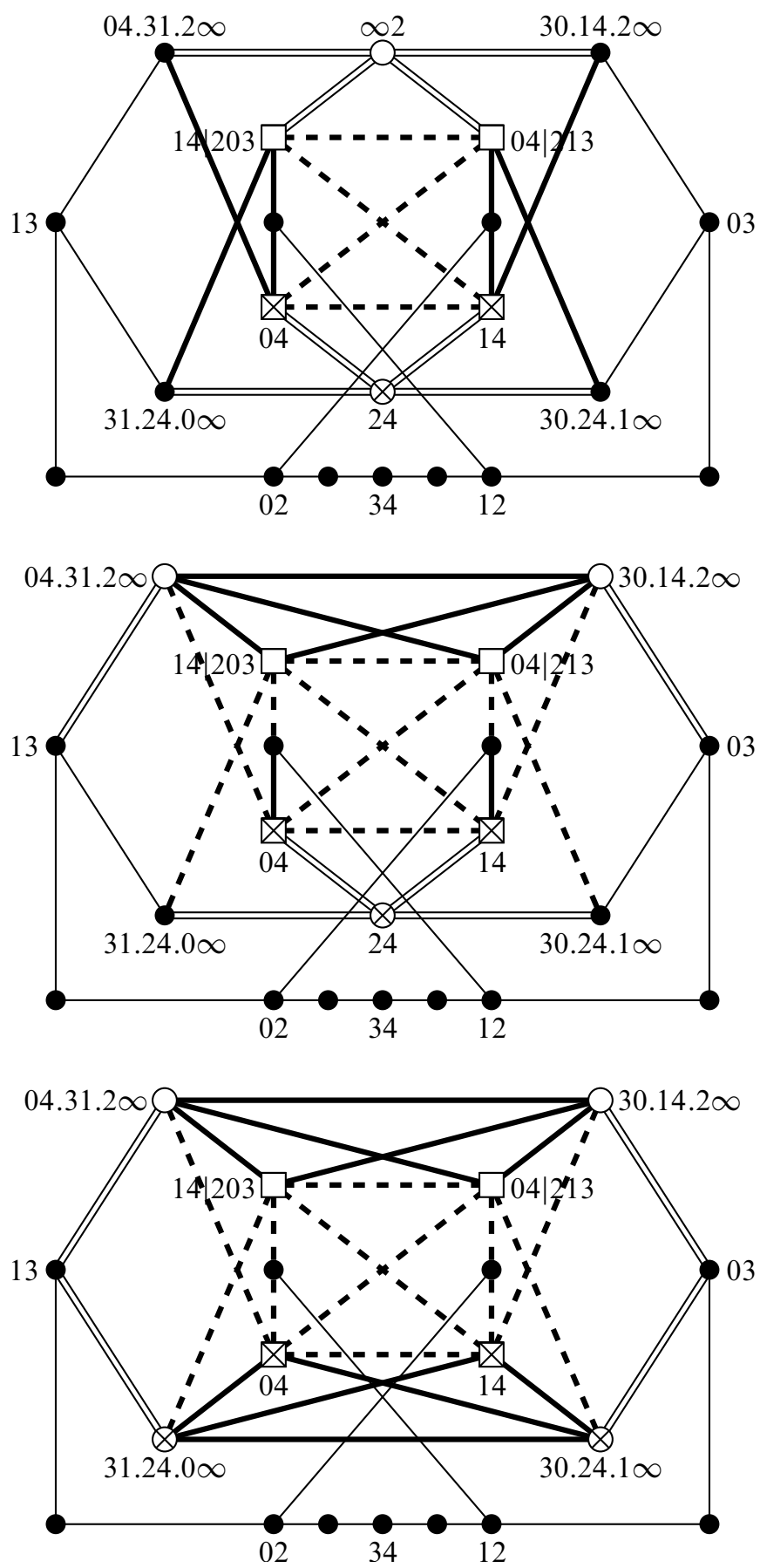

Figure 3.2: The $D_{6} D_{6}, D_{7} D_{6}$ and $D_{7} D_{7}$ faces. Left-right reflection is (01). 

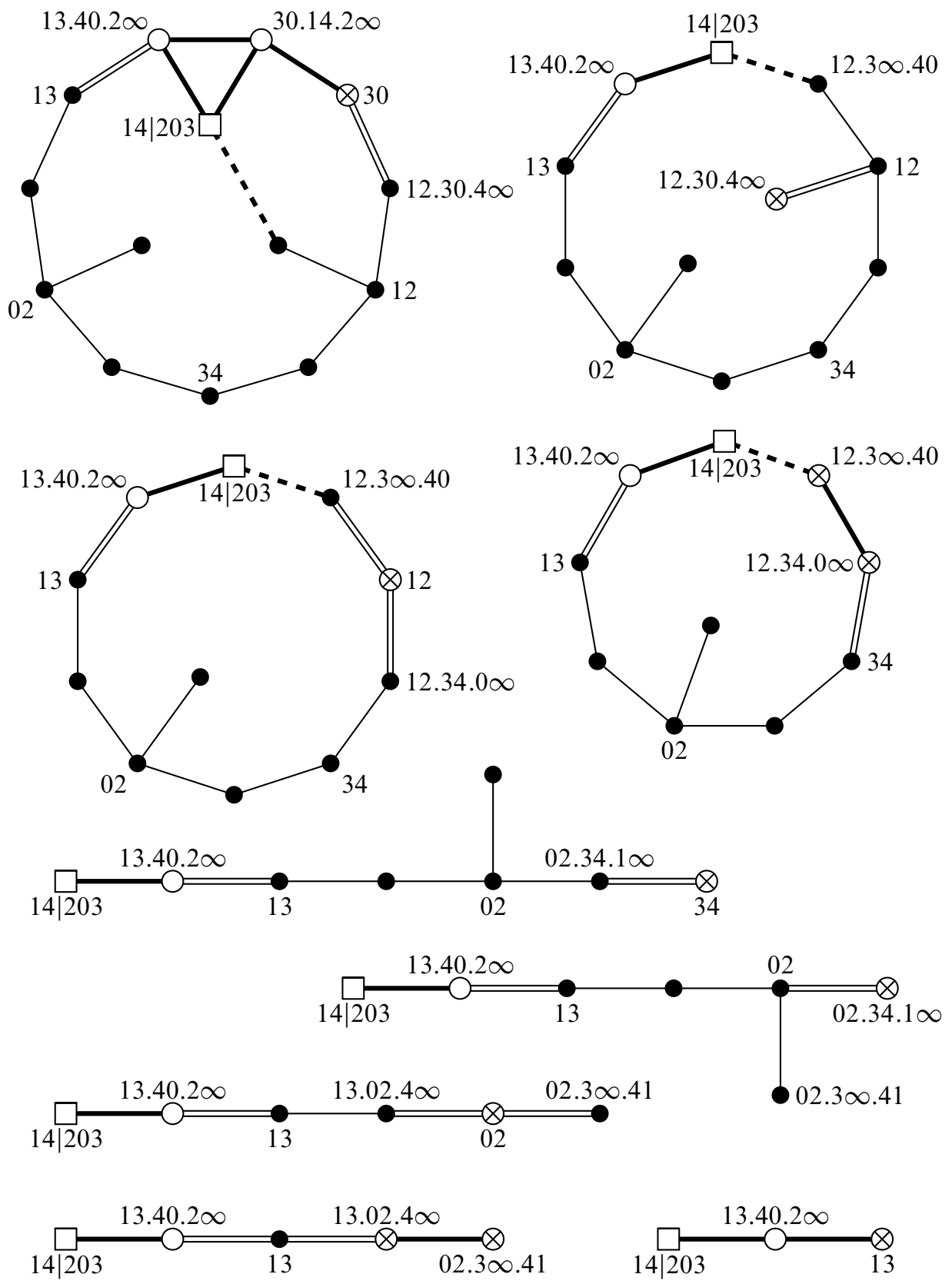

Figure 3.3: The $D_{7} D_{8}$ through $D_{7} D_{16}$ faces; for $n=12$ or 16 there are two such faces; we have chosen the $D_{7} D_{n}$ that admits an extension to $D_{7} D_{n+1}$. 
by adjoining $\infty 2$. Then we successively extend $D_{7} D_{6}$ to $D_{7} D_{7}, \ldots, D_{7} D_{16}$ by adjoining $24,30.24 .1 \infty, 30,12.30 .4 \infty, 12,12.34 .0 \infty, 34,02.34 .1 \infty, 02$ and finally $02.13 .4 \infty$. For each of these $D_{m} D_{n}$ diagrams we found the subgraph of $\Lambda$ consisting of its spherical extensions and applied Theorem 2.2 to obtain the Coxeter diagrams of the corresponding faces of $P$. The results appear in figures 3.1-3.3. The role of each extension is indicated by the nodes of the graph, according to the following scheme:

$$
\begin{array}{ll}
\bullet & D_{m} D_{n} \rightarrow D_{m} D_{n} A_{1} \\
\bigcirc & D_{m} D_{n} \rightarrow D_{m+1} D_{n} \\
\square & D_{m} D_{n} \rightarrow E_{m+1} D_{n} \\
\otimes & D_{m} D_{n} \rightarrow D_{m} D_{n+1} \\
\bigotimes & D_{m} D_{n} \rightarrow D_{m} E_{n+1}
\end{array}
$$

Nodes not named on the diagrams represent synthemes; which synthemes they are can be determined from the arrangement of duads.

We carried out the entire calculation by hand, and then wrote a computer program to repeat the calculation as a check; it corrected three minor errors, due to miscopying and the like. We made the comparison after typesetting, to avoid typographical errors.

The subgroups of $S_{5}$ acting on the various faces are described in the captions. We also remark that in the $D_{6} D_{4}$ and $D_{7} D_{4}$ faces of Figure 3.1, the odd elements of $S_{5}$ induce the diagram automorphisms of $D_{6}$ and $D_{7}$, and the permutations of 0,1 and 4 induce the diagram automorphisms of $D_{4}$. In the $D_{6} D_{6}, D_{7} D_{6}$ and $D_{7} D_{7}$ diagrams, the only element of $S_{5}$ acting is (01), which induces the diagram automorphisms of both $D_{m}$ and $D_{n}$. The additional symmetries of the $D_{6} D_{6}$ and $D_{7} D_{7}$ faces arise from elements of $C o_{\infty}$ exchanging the two $D_{m}$ components. Finally, the $D_{7} D_{11}$ face has a symmetry not induced by a symmetry of $P$.

The existence of the various diagram automorphisms proves that $\Lambda$ has a unique orbit of $D_{m} D_{n}$ diagrams for each $(m, n)$ considered here, except for $D_{7} D_{12}$ and $D_{7} D_{16}$, for which there are two orbits. The $D_{7} D_{12}$ and $D_{7} D_{16}$ diagrams we treat are those admitting extensions to $D_{7} D_{13}$ and $D_{7} D_{17}$.

\section{Variations on doubling}

Iterated doubling of redoublable polyhedra is not the only way to construct infinitely many Coxeter polyhedra. Suppose $Q$ is a Coxeter polyhedron in $H^{n}, W=W(Q)$, $w_{1}, \ldots, w_{k}$ are pairwise disjoint doubling walls, and $W_{0}$ is the subgroup of $W$ generated by the reflections $R_{1}, \ldots, R_{k}$ across them. By disjointness of the $w_{i}, W_{0}$ is a $k$-fold free product of $(\mathbb{Z} / 2)$ 's, and its Cayley graph $\Gamma$ with respect to the generators 
$R_{i}$ is a tree of valence $k$. The $W_{0}$-translates of $Q$ correspond to the vertices of $\Gamma$, with two translates disjoint unless they correspond to adjacent vertices of $\Gamma$, in which case they meet along a $W_{0}$-translate of one of the $w_{i}$.

Theorem 4.1 Suppose $T$ is any subtree of $\Gamma$ and $Q_{T}$ is the union of the translates of $Q$ corresponding to vertices of $T$. Then $Q_{T}$ is a Coxeter polyhedron.

Proof As in Lemma 1.3, every dihedral angle of $Q_{T}$ is either a dihedral angle of $Q$ or twice a dihedral angle of $Q$ that involves one of the $w_{i}$.

Corollary 4.2 Suppose $Q$ is redoublable and $I$ is any positive integer. Then $W$ has a subgroup of index $I$ which is generated by reflections.

Proof The redoublability hypothesis says we may take $k \geq 2$, so $\Gamma$ is infinite. Choose any subtree with $I$ vertices and apply the theorem.

Theorem 4.3 Suppose $Q$ has finite volume and has three or more pairwise disjoint doubling walls. Let $N(I)$ be the number of subgroups of $W$ of index $I$ that are generated by reflections, up to conjugacy by isometries of $H^{n}$. Then $N(I)$ is bounded below by an exponential in $I$.

Proof of Theorem 1.2, given Theorem 4.3 For $n=1$ there is a continuous family of compact Coxeter polyhedra, and for $n=2$ there are continuous families both of compact and noncompact Coxeter polyhedra of finite volume. We will exhibit a noncompact (resp. compact) finite-volume Coxeter polyhedron $Q$ in $H^{n}$ for $n=3, \ldots, 15,18$ and 19 (resp. $n=3, \ldots, 6$ ), with three pairwise disjoint doubling walls. Then we just apply Theorem 4.3 .

We treat the noncompact case first. For $n=19,18,15$ or 14 we take $Q$ to the $D_{6}$, $D_{7}, D_{6} D_{4}$ or $D_{7} D_{4}$ face of Conway's polyhedron $P$, the doubling walls being any three dryads. See Figure 3.1 for the diagrams for the last two of these $Q$. We will come back to $n=13$ in a moment. For $n=12,11$ or 10 we take $Q$ to be the $D_{7} D_{6}, D_{7} D_{7}$ or $D_{7} D_{8}$ face of $P$, the doubling walls being (for example) $04.31 .2 \infty, 30.14 .2 \infty$ and $14 \mid 203$. See figures 3.2 and 3.3. Returning to $n=13$, observe in Figure 3.2 that the $D_{6} D_{6}$ face of $P$ (call it $F$ ) does not have three disjoint doubling walls. Nevertheless, we can take $Q$ to be the double of $F$ across its doubling wall 14|203. Then 04, 04|213 and $\overline{04 \mid 213}$ give three disjoint doubling walls of $Q$, where the overline indicates the image of $04 \mid 213$ under the reflection used for doubling $F$.

For $n=9$ we run into the problem that the $D_{7} D_{9}$ face (Figure 3.3) does not have three disjoint doubling walls, and the doubling trick we used for $n=13$ doesn't help. 
But there is a $D_{6} D_{6} D_{4}$ face of $P$, call it $F$, which can be doubled to build a suitable $Q$. We take $F$ to be the $D_{6} D_{6} D_{4}$ face of $P$ obtained from the $D_{6} D_{6}$ face of Section 3 by taking the $D_{4}$ diagram to consist of the duad 13 and its neighboring synthemes. The Coxeter diagram for $F$ appears in Figure 4.1; we found it by using Theorem 2.2. We use the notation of Section 3, and the node $\odot$ indicates the unique extension $D_{6} D_{6} D_{4} \rightarrow D_{6} D_{6} D_{5}$ in $\Lambda$. We take $Q$ to be the double of $F$ across its doubling wall $04 \mid 213$; its diagram also appears in Figure 4.1. For the doubling walls of $Q$ we take $14,02.3 \infty .14$ and $\overline{02.3 \infty .14}$. The overline has the same meaning as before.
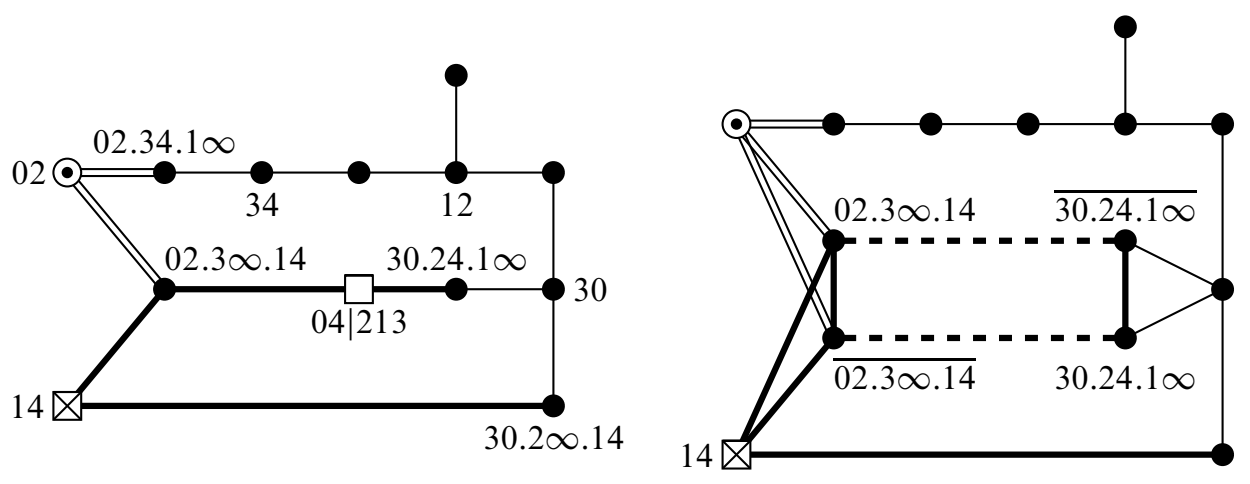

Figure 4.1: A $D_{6} D_{6} D_{4}$ face of Conway's polyhedron $P$, and its double across its wall $04 \mid 213$.

For $n=3, \ldots, 8$ we use the $n$-dimensional right-angled polyhedron from [12]. For $n=6,7$ and 8 it has three disjoint doubling walls, so we can use it for $Q$. For $n=3,4$ and 5 it does not, but after a few random doublings one finds a right-angled polyhedron with three disjoint doubling walls, which we can take for $Q$.

Now we construct our compact polyhedra. For $n=3$ (resp. 4) we take $Q$ to be the right-angled dodecahedron (resp. the right-angled 120-cell). For $n=6$ we take $Q$ to be Bugaenko's polyhedron, described in detail in Section 2. Writing $Q_{i}(i=1, \ldots, 34)$ for the walls of $Q$, in the order given, $Q_{9}, Q_{19}$ and $Q_{25}$ are pairwise disjoint doubling walls. For $n=5$ we take the wall $Q_{7}$. It is easy to see that any doubling wall of a Coxeter polyhedron is itself a Coxeter polyhedron, and it follows that $Q_{7}$ is a Coxeter polyhedron. Writing $Q_{i, j}$ for $Q_{i} \cap Q_{j}$, one can check that $Q_{7}$ has 27 walls, of which $Q_{7,9}, Q_{7,19}$ and $Q_{7,25}$ are pairwise disjoint doubling walls; indeed each is orthogonal to every wall of $Q_{7}$ that it meets. To see this, suppose $j=9,19$ or 25 and that $k \neq j$ is such that $Q_{7, j} \cap Q_{7, k} \neq \varnothing$; we claim that $Q_{7, j} \perp Q_{7, k}$. Since $Q_{7, j} \cap Q_{7, k} \neq \varnothing$, the subdiagram of $Q$ 's Coxeter diagram spanned by the $7 \mathrm{th}, j$ th and $k$ th nodes is spherical. Because the 7 th and $j$ th nodes are joined by a bond marked 8 , the $k$ th must be disjoined from both of them, so $Q_{k} \perp Q_{7}$ and $Q_{k} \perp Q_{j}$. It follows from 
elementary geometrical considerations that $Q_{7, j} \perp Q_{7, k}$. (We also see that the three doubling walls are disjoint.)

Finding finite-volume Coxeter polyhedra in $H^{16}$ and $H^{17}$ with three disjoint doubling walls would allow us to remove the $n \neq 16,17$ hypothesis from Theorem 1.2. We tried various constructions but nothing worked.

For the proof of Theorem 4.3 we need the concept of a quasi-isometry. If $X$ and $Y$ are metric spaces and $f: X \rightarrow Y$ is a function, not necessarily continuous, then we call $f$ a $(k, \ell)$-quasi-isometric embedding if for all $x, y \in X$ we have

$$
\frac{1}{k} d(x, y)-\ell \leq d(f(x), f(y)) \leq k d(x, y)+\ell .
$$

Here we take $k \geq 1$ and $\ell \geq 0$. We call $f$ a $(k, \ell)$-quasi-isometry if in addition every element of $Y$ lies at distance $\leq \ell$ of some point of $f(X)$. Under this condition, we may find a sort of inverse for $f$ by defining $g(y)$ to be any point of $X$ with $f(x)$ within $\ell$ of $y \in Y$. One can check that $g$ is a $(k, 3 k \ell)$-quasi-isometry. Finally, the composition of a $(k, \ell)$-quasi-isometry followed by a $\left(k^{\prime}, \ell^{\prime}\right)$-quasi-isometry is a $\left(k k^{\prime}, k^{\prime} \ell+2 \ell^{\prime}\right)$-quasi-isometry.

Lemma 4.4 For every $k \geq 1$ and $\ell \geq 0$ there exists $L>0$ such that if $T$ and $T^{\prime}$ are trees with no vertices of valence 2 , metrized such that each edge has length $\geq L$, and there is a $(k, \ell)$-quasi-isometry $f: T \rightarrow T^{\prime}$, then $T$ and $T^{\prime}$ are isomorphic as combinatorial graphs.

Sketch of proof We give the ideas, which the reader can follow to supply explicit estimates if desired. One takes $L$ to be much larger than any of the constants appearing in the argument, all of which involve only $k$ and $\ell$. Suppose $T, T^{\prime}$ and $f$ are as in the statement of the lemma. The key point is that with $a=3 k \ell$ and $L=2(k a+\ell)$, every branch point $B$ of $T$ maps to within $k a+\ell$ of exactly one branch point $B^{\prime}$ of $T^{\prime}$. To see this one considers the points $x_{i}$ (i in some index set) on the edges emanating from $B$, at distance $a$ from $B$. One argues that no $x_{i}$ can map into the segment $\left[f(B), f\left(x_{j}\right)\right]$ from $f(B)$ to $f\left(x_{j}\right)$, for $j \neq i$. Therefore none of the segments $\left[f(B), f\left(x_{i}\right)\right]$ contains any other, and this can only happen if $f(B)$ lies at distance $<k a+\ell$ of some branch point of $T^{\prime}$. Since $T^{\prime}$ has edges more than twice as long as this, $f(B)$ lies within $k a+\ell$ of exactly one branch point of $T^{\prime}$. This gives a map

$$
F:\{\text { branch points of } T\} \rightarrow\left\{\text { branch points of } T^{\prime}\right\} .
$$

Enlarging $L$, we may suppose $F$ is injective. Applying the same argument to the "inverse" quasi-isometry $g: T^{\prime} \rightarrow T$, one shows (after enlarging $L$ again) that $F$ is 
surjective. Enlarging $L$ again, one can choose $b>0$ such that each edge of $T$, minus the length $b$ segments at its ends, maps into exactly one edge of $T^{\prime}$. This gives a map from edges of $T$ to edges of $T^{\prime}$, which we also denote by $F$. Enlarging $L$ as necessary, one proves that $F$ is injective and surjective on edges and preserves the incidence relation between edges and branch points of $T$. This implies that $F$ is a graph isomorphism.

Proof of Theorem 4.3 After doubling $Q$ a few times, we may assume that $Q$ has three doubling walls which are pairwise ultraparallel. We choose a basepoint $q$ in the interior of $Q$. Let $V>0$ be small enough that the volume $V$ closed horoball neighborhoods around distinct cusps of $Q$ are disjoint. By shrinking $V$ we may suppose that the perpendiculars from $q$ to the three doubling walls miss these horoball neighborhoods. For any finite subtree $T$ of $\Gamma$ let $Q_{T}^{-}$be the subset of $Q_{T}$ obtained by deleting the volume $V$ closed horoball neighborhoods of the cusps of $Q_{T}$. (All proper subtrees of $\Gamma$ occurring in this proof are finite; we will omit explicit mention of this.) By joining translates of $q$ by geodesics when they lie in neighboring $W_{0}$-translates of $Q$, we may regard $\Gamma$ as embedded in $H^{n}$ (denote the embedding by $i$ ), and in fact $T$ is embedded in $Q_{T}^{-}$.

We claim that there exist $k \geq 1$ and $\ell \geq 0$ such that for all $T, i: T \rightarrow Q_{T}^{-}$is a $(k, \ell)$-quasi-isometry, where $\Gamma$ is equipped with the metric in which edges have unit length, and $Q_{T}^{-}$is equipped with its natural path metric. To see this we begin by observing that $i: \Gamma \rightarrow H^{n}$ is a $(k, \ell)$-quasi-isometric embedding for some $(k, \ell)$; this is a consequence of the fact that the doubling walls are ultraparallel. In fact, $W_{0}$ is a Fuchsian group, preserving the unique $H^{2}$ orthogonal to the three doubling walls, with the generating reflections acting on it by reflections across three pairwise ultraparallel lines. We enlarge $k$ if necessary so that every edge of $i(\Gamma)$ has length $\leq k$. Now, for any $T$ and $x, y \in T$, we have

$$
\begin{aligned}
\frac{1}{k} d_{T}(x, y)-\ell \leq d_{H^{n}}(i(x), i(y)) & \leq d_{Q_{T}^{-}}(i(x), i(y)) \\
& \leq d_{i(T)}(i(x), i(y)) \leq k d_{T}(x, y),
\end{aligned}
$$

and it follows that $i: T \rightarrow Q_{T}^{-}$is a $(k, \ell)$-quasi-isometric embedding. By enlarging $\ell$ we may suppose that for every $T$, every point of $Q_{T}^{-}$lies within $\ell$ of some point of $i(T)$. To do this, take $\ell$ at least as large as the diameter of the subset of $Q$ obtained by deleting the volume $V / 2$ horoball neighborhoods of the cusps of $Q$. (The factor of $1 / 2$ comes from the fact that a cusp of $Q_{T}$ may be a cusp of two different $W_{0}$-translates of $Q$. A cusp of $Q_{T}$ cannot be a cusp of more than two $W_{0}$-translates of $Q$, because the doubling walls are ultraparallel.) We have proven our claim. 
Now, suppose $T$ and $T^{\prime}$ are subtrees of $\Gamma$ with $Q_{T}$ and $Q_{T^{\prime}}$ isometric. Then $Q_{T}^{-}$ and $Q_{T^{\prime}}^{-}$are isometric. Since $T \rightarrow Q_{T}^{-}$and $T^{\prime} \rightarrow Q_{T^{\prime}}^{-}$are $(k, \ell)$-quasi-isometries, there is a $\left(k^{2}, 7 k \ell\right)$-quasi-isometry $T \rightarrow T^{\prime}$. Plugging $\left(k^{2}, 7 k \ell\right)$ into Lemma 4.4, we obtain $L>0$ with the properties stated there.

Consider $I$-vertex subtrees $T$ of $\Gamma$ for which the branch points of $T$ lie at distance $\geq L$ in $\Gamma$. If two such trees are not isomorphic as abstract graphs, then their corresponding polyhedra cannot be isometric. The number of isomorphism classes of abstract trivalent trees with up to $\left\lfloor\frac{I-1}{L}\right\rfloor$ edges is bounded below by an exponential in $\left\lfloor\frac{I-1}{L}\right\rfloor$ and hence by an exponential in $I$. ( $\lfloor x\rfloor$ means the largest integer $\leq x$.) Therefore we may choose for each $I \geq 1$ a set $\mathcal{T}_{I}$ of $I$-vertex subtrees of $\Gamma$, with distinct elements of $\mathcal{T}_{I}$ giving non-isometric polyhedra, and $\left|\mathcal{T}_{I}\right|$ growing exponentially with $I$.

\section{References}

[1] R Borcherds, Automorphism groups of Lorentzian lattices, J. Algebra 111 (1987) 133-153 MR913200

[2] R E Borcherds, Coxeter groups, Lorentzian lattices, and K3 surfaces, Internat. Math. Res. Notices (1998) 1011-1031 MR1654763

[3] V O Bugaenko, Groups of automorphisms of unimodular hyperbolic quadratic forms over the ring $\mathbf{Z}[(\sqrt{5}+1) / 2]$, Vestnik Moskov. Univ. Ser. I Mat. Mekh. (1984) 6-12 MR764026

[4] V O Bugaenko, On reflective unimodular hyperbolic quadratic forms, Selecta Math. Soviet. 9 (1990) 263-271 MR1074386

[5] V O Bugaenko, Arithmetic crystallographic groups generated by reflections, and reflective hyperbolic lattices, from: "Lie groups, their discrete subgroups, and invariant theory”, Adv. Soviet Math. 8, Amer. Math. Soc., Providence, RI (1992) 33-55 MR1155663

[6] J H Conway, N J A Sloane, Leech roots and Vinberg groups, Proc. Roy. Soc. London Ser. A 384 (1982) 233-258 MR684311 Reprinted as ch. 28 of [7]

[7] J H Conway, N J A Sloane, Sphere packings, lattices and groups, Grundlehren series 290, Springer, New York (1993) MR1194619

[8] F Esselmann, The classification of compact hyperbolic Coxeter $d$-polytopes with $d+2$ facets, Comment. Math. Helv. 71 (1996) 229-242 MR1396674

[9] I M Kaplinskaja, The discrete groups that are generated by reflections in the faces of simplicial prisms in Lobačevskĭ spaces, Mat. Zametki 15 (1974) 159-164 MR0360858

[10] VS Makarov, The Fedorov groups of four-dimensional and five-dimensional Lobačevskil space, from: "Studies in General Algebra, No. 1 (Russian)", Kišinev. Gos. Univ., Kishinev (1968) 120-129 MR0259735 
[11] PARI/GP, version 2.1.5, http://pari.math.u-bordeaux.fr/

[12] L Potyagailo, E Vinberg, On right-angled reflection groups in hyperbolic spaces, Comment. Math. Helv. 80 (2005) 63-73 MR2130566

[13] M N Prokhorov, Absence of discrete groups of reflections with a noncompact fundamental polyhedron of finite volume in a Lobachevskiŭ space of high dimension, Izv. Akad. Nauk SSSR Ser. Mat. 50 (1986) 413-424 MR842588

[14] O P Ruzmanov, Examples of nonarithmetic crystallographic Coxeter groups in $n$ dimensional Lobachevskiŭ space when $6 \leq n \leq 10$, from: "Problems in group theory and in homological algebra (Russian)", Yaroslav. Gos. Univ., Yaroslavl' (1989) 138-142 MR1068774

[15] P V Tumarkin, Compact hyperbolic Coxeter $n$-polytopes with $n+3$ facets arXiv: math. MG/0406226

[16] PV Tumarkin, Hyperbolic Coxeter polytopes in $\mathfrak{H}^{m}$ with $n+2$ hyperfacets, Mat. Zametki 75 (2004) 909-916 MR2086616

[17] P V Tumarkin, Hyperbolic $n$-dimensional Coxeter polytopes with $n+3$ facets, Tr. Mosk. Mat. Obs. 65 (2004) 253-269 MR2193442

[18] ̇ B Vinberg, The groups of units of certain quadratic forms, Mat. Sb. (N.S.) 87(129) (1972) 18-36 MR0295193

[19] ̀ B Vinberg, The nonexistence of crystallographic reflection groups in Lobachevskĭ spaces of large dimension, Funktsional. Anal. i Prilozhen. 15 (1981) 67-68 MR617472

[20] È B Vinberg, The two most algebraic K3 surfaces, Math. Ann. 265 (1983) 1-21 MR719348

[21] È B Vinberg, I M Kaplinskaja, The groups $O_{18,1}(Z)$ and $O_{19,1}(Z)$, Dokl. Akad. Nauk SSSR 238 (1978) 1273-1275 MR0476640

Department of Mathematics, University of Texas at Austin

Austin, TX 78712, USA

allcock@math.utexas.edu

http://www. math.utexas.edu/ allcock

Proposed: Martin Bridson

Received: 28 April 2005

Seconded: Benson Farb, Walter Neumann

Revised: 8 August 2005 